\title{
Utility of a Genomic-based, Personalized Medicine Test in Patients Presenting With Symptoms Suggesting Coronary Artery Disease
}

\author{
Lee Herman, MD, James Froelich, MD, Dino Kanelos, MD, Robert St. Amant, MD, \\ May Yau, MS, Brian Rhees, PhD, Mark Monane, MD, and Jobn McPherson, MD
}

Purpose: Better methods are needed to assess patients presenting with symptoms suggestive of obstructive coronary artery disease (CAD). We hypothesized that the use of a gene expression score (GES) would lead to a change in the diagnostic evaluation.

Methods: The Primary Care Providers Use of a Gene Expression Test in Coronary Artery Disease Diagnosis (IMPACT-PCP) trial (clinical trial identifier NCT01594411, clinicaltrials.gov) was a prospective study of stable, nonacute, nondiabetic patients presenting with chest pain and related symptoms at 4 primary care practices. All patients underwent GES testing, with clinicians documenting their planned diagnostic strategy both before and after GES. The GES was derived from a peripheral blood draw measuring expression of 23 genes and has been shown to have a $96 \%$ negative predictive value for excluding the diagnosis of obstructive CAD.

Results: Of the 251 study patients, 140 were women (56\%); the participants had a mean age of 56 years (standard deviation, 13.0) and a mean body mass index of $30 \mathrm{mg} / \mathrm{kg}^{2}$ (standard deviation, 6.7). The mean GES was 16 (range, 1-38), and 127 patients (51\%) had a low GES ([ltqeu]15). A change in the diagnostic testing pattern before and after GES testing was noted in 145 of 251 patients $(58 \% \mathrm{ob}$ served vs. $10 \%$ predefined expected change; $P<.001)$.

Conclusions: Incorporation of the GES into the diagnostic workup showed clinical utility above and beyond conventional clinical factors by optimizing the patient's diagnostic evaluation. ( $\mathrm{J}$ Am Board Fam Med 2014;27:258-267.)

Keywords: Cardiovascular Abnormalities, Genetics, Medical Decision Making, Practice-based Research, Technology Assessment

Evaluating patients with chest pain and related symptoms to determine the current likelihood of coronary artery disease (CAD) is a common clinical

This article was externally peer reviewed.

Submitted 15 May 2013; revised 1 November 2013; accepted 11 November 2013.

From Johns Creek Primary Care, Suwanee, GA (LH); Family Care Clinic, Bonham, TX (JF); Carolina Family Healthcare, Weddington, NC (DK); The Lipid Center, Baton Rouge, LA (RS); CardioDx, Inc., Palo Alto, CA (MY, BR, MM); and Vanderbilt University, Nashville, TN (JM).

Funding: Funding was provided by CardioDx, Inc.

Prior presentation: This work was presented in part at the Quality of Care and Outcomes Research in Cardiovascular Disease and Stroke 2013 Scientific Sessions, May 15-17, 2013, Baltimore, Maryland.

Conflict of interest: LH and JM have received research support from CardioDx, Inc. MY, BR, and MM are employees of CardioDx, Inc.

Corresponding author: Lee Herman, MD, Johns Creek Primary Care, 4365 Johns Creek Pkwy, Suite 400, Suwanee, GA 30024 (E-mail: drleeherman@yahoo.com). scenario, with approximately 8000 patients presenting every day to primary care clinicians in the Unites States. ${ }^{1}$ These patients may present with typical symptoms of angina (such as substernal tightness, shortness of breath, or dyspnea on exertion), but they frequently complain of atypical symptoms (such as heartburn, fatigue, and dizziness) that have an unclear etiology. These atypical symptoms, which typically are associated with gastroesophageal reflux, anxiety, and fibromyalgia, may also represent symptoms of obstructive CAD, especially among women and the elderly. ${ }^{2-4}$

Physicians strive to integrate available clinical data (eg, clinical history and physical examination) with the results of noninvasive tests (eg, exercise electrocardiographic testing, stress echocardiography, myocardial perfusion imaging [MPI], and coronary computed tomographic angiography) to de- 
termine the likelihood of obstructive $\mathrm{CAD}$ and the clinical need to refer for additional testing. The pretest likelihood of $\mathrm{CAD}$ in symptomatic patients, as defined by scoring systems such as the Diamond/ Forrester classification and coronary angiographic scoring system data, have been developed and validated to help clinicians estimate the probability of CAD based on symptoms, age, and sex. ${ }^{5,6}$ Yet physician assessments and test results often fail to establish the cause of the patient's symptoms as well as exclude obstructive CAD. ${ }^{7}$ Despite a thorough and potentially expensive workup, only $10 \%$ of chest pain cases evaluated by physicians ultimately result in a diagnosis of obstructive CAD.$^{8-11}$ In a recently published registry of more than 14,000 patients, the pretest probability of CAD based on clinical factors in patients referred for advanced cardiovascular imaging overestimated the actual presence of disease by $57 \%$ in patients with typical angina and $32 \%$ in patients with atypical angina. ${ }^{12}$ Furthermore, results of a recent study showed abnormal findings on MPI in $<10 \%$ of patients referred for further workup of suspected obstructive CAD. ${ }^{13}$

As a consequence of this diagnostic uncertainty, the evaluation of CAD remains highly variable. Challenges with adherence to and interpretation of clinical guidelines lead to both overutilization and underutilization of imaging. ${ }^{14-17}$ This finding poses an enormous economic burden on the health care system, leading to approximately $\$ 6.7$ billion in noninvasive and invasive testing costs for nondiabetic patients with no prior revascularization or myocardial infarction initially evaluated in the primary care or cardiology setting. ${ }^{18-22}$ Furthermore, the additional risks associated with subsequent diagnostic testing include exposure to radiation, contrast-induced anaphylaxis, and acute kidney injury. ${ }^{23-25}$

Better methods are needed to more accurately assess the likelihood of obstructive CAD among stable patients presenting to primary care clinicians in an outpatient setting. This unmet need is especially relevant for patients with low to intermediate pretest probability of disease, such as women and patients who present with atypical symptoms. The recent development of a genomic-based, personalized medicine test measuring expression levels of 23 genes in white blood cells may offer a solution for this diagnostic dilemma. ${ }^{26}$ In the prospective, multicenter, blinded PREDICT and COMPASS validation studies, a low gene expression score (GES) $(\leq 15)$ had a negative predictive value and sensitivity of $83 \%$ and $85 \%$ (PREDICT) and $96 \%$ and $89 \%$ (COMPASS), respectively, for excluding the diagnosis of obstructive CAD among patients referred for further invasive and noninvasive cardiovascular testing. ${ }^{27,28}$ We hypothesized that GES results would reduce diagnostic uncertainty in the evaluation of stable patients presenting in the primary care setting with symptoms suggestive of obstructive CAD and lead to a change in the medical decision making, including the need to refer for further cardiac testing.

\section{Methods}

\section{Setting and Participants}

The study group included 261 stable, nonacute, nondiabetic patients who presented to primary care clinicians for the evaluation of suspected obstructive CAD and underwent GES testing from April 2012 to January 2013. The study sites included 9 clinicians at 4 community-based primary care practices in Suwanee, Georgia (site 1); Weddington, North Carolina (site 2); Baton Rouge, Louisiana (site 3); and Bonham, Texas (site 4). With respect to the demographics of the primary care clinicians, there were 5 physicians (average experience, 23 years; range, 13-35 years) and 4 nonphysicians (2 nurse practitioners and 2 physician assistants; average experience, 14 years; range, 12-16 years). Primary care physicians, nurse practitioners, and physician assistants at these primary care practices were educated and trained in the use and interpretation of the GES through a standardized in-service program. This training comprised $\sim 5$ hours and included all the appropriate personnel in the clinicians' office: nurse, phlebotomist, office manager, and others as appropriate. In addition, there was an additional 1 hour of training about the research protocol for this trial. The GES test is intended for patients with a history of chest pain, with suspected anginal equivalents to chest pain, or with a high risk of CAD. Patients experiencing acute coronary syndrome (ie, myocardial infarction or unstable angina) or patients who require urgent evaluation are not appropriate patients for the GES test. The GES is not intended for use in patients with diabetes, systemic infectious or systemic inflammatory conditions, or who are currently taking steroids, immunosuppressive agents, or chemotherapeutic 
agents. Patients were eligible for this study if they met the GESs' intended use criteria and had symptoms equivalent to chest pain or angina. Clinicians were allowed to incorporate GES testing at their own discretion as part of their clinical decisionmaking processes.

\section{Description of the GES}

The GES (Corus CAD; CardioDx, Inc., Palo Alto, CA) was calculated by a commercially available, Medicare-covered, validated, quantitative diagnostic test that measures expression levels of 23 genes in 6 terms by quantitative reverse transcriptase polymerase chain reaction from a peripheral blood sample to determine a patient's likelihood of obstructive CAD (one or more coronary arteries with $\geq 50 \%$ angiographic stenosis, as determined by quantitative coronary angiography or core-lab computed tomography/angiography). ${ }^{27,28}$ Whereas genetic testing provides a prediction of lifetime disease risk, the genomic-based GES test provides an assessment of the current state of obstructive CAD by measuring gene expression changes associated with atherosclerosis. The GES algorithm comprises expression values for 23 genes from peripheral blood cells in 6 terms, patient age, and sex. Each term is composed of ratios of highly correlated genes representing a diverse set of inflammatory cell biology, including neutrophil apoptosis, neutrophil-to-lymphocyte ratio, and natural killer cell activation. These genes are selectively expressed in multiple types of circulating cells, including neutrophils (eg, caspase-5 and S100A12), natural killer cells (SLAMF7 and KLRC4), as well as $\mathrm{B}$ and $\mathrm{T}$ lymphocytes. These cells play supporting roles for both adaptive and innate immune responses in atherosclerosis. Furthermore, there are both sex-specific and common algorithm terms with sex-specific weights.

The changes in gene expression are quantified using an algorithm that generates a GES ranging from 1 to 40. A score $<15$ indicates a low risk of underlying obstructive coronary disease. ${ }^{28}$ The GES has been shown to have a negative predictive value of $96 \%$ for GES $\leq 15$ in a population referred to MPI.

\section{Study Design}

In this prospective trial (Investigation of a Molecular Personalized Coronary Gene Expression Test on Primary Care Practice Pattern, clinical trial identifier NCT01594411), the primary care clinicians initially determined patients' pretest probability for CAD based on risk factors, assessment of clinical symptoms, and results of any prior electrocardiographic stress testing. In parallel with the clinicians' pretest probability assessment, the Diamond-Forrester questionnaire was administered to evaluate the quality of the patients' anginal symptoms. Symptoms were classified as typical angina, atypical angina, or noncardiac pain based on the presence of one or more typical symptoms-substernal chest discomfort, aggravation upon exertion, and alleviation with rest-as well as atypical symptoms such as heartburn, palpitations, malaise, and fatigue. ${ }^{29}$

A clinical report form captured the clinicians' preliminary clinical assessments and impressions ("preliminary decision"), including recommendations for further evaluation and management of the patient. Peripheral blood samples were collected simultaneously in the outpatient setting into a vial of RNA preservation fluid (PAXgene RNA Blood Tubes; PreAnalytix, Valencia, CA) according to the manufacturer's instructions and shipped to a single clinical laboratory certified by the Clinical laboratory Improvement Amendments and accredited by the College of American Pathology laboratory (CardioDx, Inc., Palo Alto, CA; College of American Pathology laboratory no. 8646908), which reported the GES to the primary care provider within 2 to 3 days, on average. After receiving the GES, the physicians completed the clinical report form by entering the final clinical assessment and impression ("final decision"), using the GES results to support or modify the initial clinical decision.

The primary objective of the study was to assess whether the use of the GES altered the clinicians' evaluations, as defined by a change in patient management between the preliminary and final decision. This change was prospectively defined as either a downgrade or upgrade in the intensity of the diagnostic plan based on the following categories (in hierarchical order): (1) no further cardiac testing or treatment, (2) lifestyle changes or medical therapy, (3) stress testing (with or without imaging) or computed tomography/coronary angiography, or (4) invasive coronary angiography. Secondary analyses assessed the patterns of change (decrease or increase in diagnostic intensity) for each patient. To assess the effects of changing the diagnostic approach, as well as the effect these changes had on 
patient outcomes, for each enrolled patient we conducted a follow-up phone call or chart review at least 30 days after the GES testing. We collected information during the follow-up assessment to determine whether the final diagnostic plan was followed, which cardiac diagnostic tests and procedures were performed, and the results of these tests and procedures. Major adverse cardiac event data also were recorded over the ensuing 30 days.

The local hospital institutional review board or central institutional review board (Quorom, Inc.) approved the study, and all patients submitted written informed consent. Prespecified data, including demographics, clinical risk factors, symptoms, medical history, medications, prior testing results, and GES results, were obtained by research study coordinators using standardized data collection methods. Data were verified by independent study monitors.

\section{Statistical Analysis}

For the analysis of the primary end point, changes in the management plan between the patients' preliminary and final evaluations were reviewed. The proportion of patients whose preliminary and final treatment decision differed was compared using a one-sided binomial test to $10 \%$, which is the minimum amount believed to be clinically relevant.

To assess whether there was a trend toward decreased or increased treatment intensity, treatments before and after GES were ranked by their intensity $(1=$ no further cardiac testing or treatment; 2 = lifestyle changes or medical therapy for angina or noncardiac chest pain; $3=$ stress testing with or without imaging or computed tomography/ coronary angiography; 4 = invasive cardiac catheterization). The counts of patients whose testing plans were decreased or increased in intensity were compared using a sign test (exact 2 -sided binomial) to determine whether the frequency of increased management plans was equal to those that were decreased. Treatment plans before and after GES were tested for independence from the GES using exact tests of association.

Standard statistical methods were used for all comparisons, with an $\alpha$ level of $0.05 .^{30}$ All analyses were performed using $\mathrm{R}$ software version 2.15 (including rms and reshape2 packages; available at http://www.r-project.org/). In cases where patient records were incomplete, missing data frequencies were estimated and reported, with calculations based on nonmissing values.

\section{Results}

The study enrolled 261 consecutive stable, nonacute, nondiabetic patients presenting with typical and atypical symptoms of obstructive CAD; 251 patients were eligible for primary end point analysis. Among the 10 patients who were excluded, 7 had GES exclusionary criteria (chronic inflammatory disease or diabetes) and 3 provided additional diagnostic information to the clinician after the GES blood draw (Figure 1). The study cohort (Table 1) included 140 women (56\%); the cohort had an overall mean age $56 \pm 13$ years and a mean $\mathrm{BMI}$ of $30 \pm 6.7$ $\mathrm{kg} / \mathrm{m}^{2}$. The mean GES was $16 \pm 10$ (range, $1-38$ ); 127 patients $(51 \%)$ had a low GES $(\leq 15)$.

Among the 251 patients, 154 (61\%) presented with chest pain and were classified using the Diamond-Forrester questionnaire as having typical angina $(\mathrm{n}=31)$, atypical angina $(\mathrm{n}=67)$, and noncardiac chest pain $(\mathrm{n}=56)$. Another 50 patients (20\%) presented with shortness of breath, while the remaining 47 patients (19\%) presented with atypical symptoms such as malaise/fatigue and dizziness. The average pretest probability of obstructive $\mathrm{CAD}$ as determined by the primary care clinician was $28 \pm 17 \%$. Hypertension and dyslipidemia were present in $48 \%(\mathrm{n}=120)$ and 59\% $(\mathrm{n}=147)$ of patients, respectively.

The primary outcome demonstrated a change in the diagnostic plan (ie, change between the preliminary and final decision) following GES testing in $58 \%$ of patients ( $\mathrm{n}=145 ; P<.001$, lower bound $52 \%)$. The change rates were similar $(56 \%$ and $63 \% ; P=$ nonsignificant) among physicians and nonphysicians, respectively. Of the 145 patients who had a change in diagnostic plan, $57 \%(\mathrm{n}=83)$ were female. In the preplanned secondary analyses, more patients showed a reduction $(\mathrm{n}=93)$ than an increase $(\mathrm{n}=52)$ in intensity of testing $(P<.001)$. Specifically, $82 \%$ of patients with decreased testing (76 of 93) had a low GES, whereas $94 \%$ of patients with increased testing (49 of 52) had an elevated GES $(P<.001)$. The greatest change in testing intensity was seen among patients with a low GES: $60 \%$ of patients (76 of 127 ) had reduced testing, and the majority of the remaining patients with a low GES (48 of 127) had no change in testing (Table 2). Among the 53 patients assigned to stress testing before GES and who subsequently were determined to have a low GES, only 5 patients (9\%) were referred for stress testing after GES 
Figure 1. Patient screening, enrollment, and follow-up flowchart. CAD, coronary artery disease; GES, gene expression score.

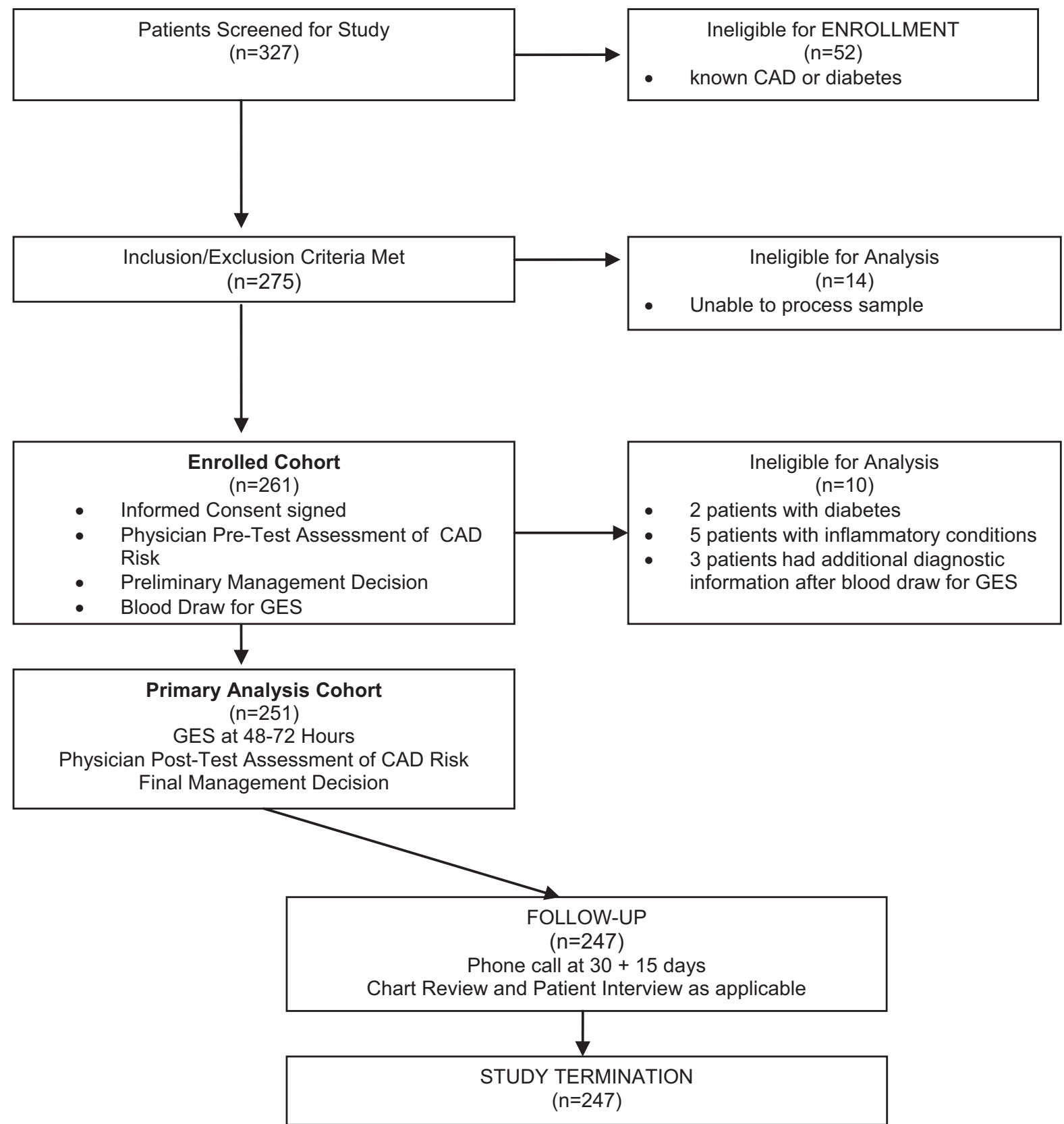

(Figure 2). A similar directional trend around change in diagnostic testing was seen among patients with an elevated GES. Of the 65 patients assigned to stress testing before GES, all were determined to have an elevated GES. An additional 34 patients with an elevated GES were assigned to stress testing after GES.

Of the 247 patients with at least 30 days of follow-up, $1(0.4 \%)$ had a major adverse cardiovascular event (hemorrhagic stroke in a patient with a low GES 5 days after GES testing, judged by the clinician investigator to be not related to the study protocol). Comparing the completed diagnostic testing with the final treatment plan decision demonstrated high clinician compliance with the patient care decision after the GES. The analysis showed an $85 \%$ agreement rate (209 of $247 \mathrm{pa}-$ tients) between the 2 scenarios.

\section{Discussion}

Our study demonstrated the clinical utility of the GES among patients presenting to the primary care 
Table 1. Clinical Characteristics of the Study Patients $(\mathrm{n}=251)$

\begin{tabular}{lr}
\hline Characteristics & Patients \\
\hline Clinical factors & \\
Female sex & $140(56)$ \\
Age (years) & $56.2 \pm 13$ \\
Non-Hispanic white ethnicity & $224(89)$ \\
Presenting symptoms & \\
Typical angina & $31(12)$ \\
Atypical angina & $67(27)$ \\
Noncardiac chest pain & $56(22)$ \\
Shortness of breath & $50(20)$ \\
Malaise/fatigue & $47(19)$ \\
Cardiac risk factors & \\
Hypertension & $120(48)$ \\
Dyslipidemia & $146(58)$ \\
Body mass index & $29.7 \pm 6.7$ \\
Systolic/diastolic blood & $128 / 79$ \\
pressure (mm Hg) & \\
Gene expression score & $16.0 \pm 10.0$ \\
\hline
\end{tabular}

Data are $\mathrm{n}(\%)$ or mean \pm standard deviation unless otherwise indicated.

clinician with typical and atypical symptoms suggestive of obstructive CAD. The GES was associated with a statistically significant and clinically relevant change in the diagnostic workup in $58 \%$ of patients. We observed that the GES was associated with a decrease in intensity of testing in $60 \%$ (76 of 127) of patients with a low GES. These findings address a major issue in the nexus of primary care: the clinically challenging diagnosis and management of obstructive CAD-a dilemma that is faced by primary care physicians on almost a daily basis. ${ }^{31}$

We note 3 areas of added interest in these findings. First, primary care clinicians successfully incorporated the use of this genomic-based, personalized medicine gene expression test into the office setting with a minimal amount of training. Clinicians in the trial noted that the 2- to 3-day turnaround time for the GES was sufficient to allow them to make informed clinical decisions for patient care. Utilization of the GES allowed physicians to reclassify patients for subsequent testing, improving on usual care while potentially reducing patient exposure to ionizing radiation. These results were similar to those seen in another study of 83 prospective patients with chest pain and related symptoms referred to cardiology for workup. ${ }^{32}$ In that study, a change in diagnostic testing occurred in $58 \%$ of patients $(\mathrm{n}=48 ; P<.001)$ following
GES testing. Of note, 91\% (29 of 32) of patients with decreased testing in the cardiology office had low GES ( $\leq 15$ ), whereas $100 \%$ (16 of 16 ) of patients with increased testing had an elevated GES $(P<.001)$.

Second, the before/after study design allowed the patient to act as his or her own control when comparing the 2 decisions, adding a robust quality to the change elicited in the diagnostic plan as a result of GES testing. For comparison, a recent study demonstrated that the incorporation of an $\mathrm{N}$-terminal prohormone of brain natriuretic peptide level in a diagnostic prediction model for acute heart failure resulted in reclassification of $44 \%$ of patients to either low- or high-probability categories. ${ }^{33}$ Other molecular diagnostic tests, most commonly in oncology, have noted similar success. In a study focusing on optimizing breast cancer treatment, results from a 21-gene recurrence score assay led medical oncologists to change the treatment recommendation for $31 \%$ of patients. ${ }^{34}$ Another analysis evaluating the diagnosis and management of patients with metastatic cancer of unknown origin showed that physicians changed their primary working diagnosis for $50 \%$ of patients $(95 \%$ confidence interval, 43-58\%) after results from a 2000gene expression profiling test. ${ }^{35}$ Third, use of this office-based GES test was not associated with untoward outcomes within the first 30 days of followup: only a $0.4 \%$ rate of major adverse cardiovascular events was detected.

Clinician-driven directional changes in diagnostic testing intensity, associated with both low as well as elevated GESs, were noteworthy in this study. We found that $82 \%$ of patients (76 of 93) with decreased intensity of testing had a low GES, whereas $94 \%$ of patients (49 of 52) with increased intensity of testing had an elevated GES. These clinical decisions are supported by the COMPASS

Table 2. Association of Testing Changes Among Patients with Low and Elevated Gene Expression Scores

\begin{tabular}{lccr}
\hline & \multicolumn{2}{c}{ Score Class } & \\
\cline { 2 - 3 } Testing Intensity & \multicolumn{1}{c}{$\leq 15$} & $>15$ & All \\
\hline Decreased & $76(60)$ & $17(14)$ & 93 \\
No change & $48(38)$ & $58(47)$ & 106 \\
Increased & $3(2)$ & $49(39)$ & 52 \\
All & $127(100)$ & $124(100)$ & 251 \\
\hline
\end{tabular}

Data are $\mathrm{n}(\%) . P<.00001$, exact test. 
Figure 2. Treatment decision before and after gene expression testing. GES, gene expression score.

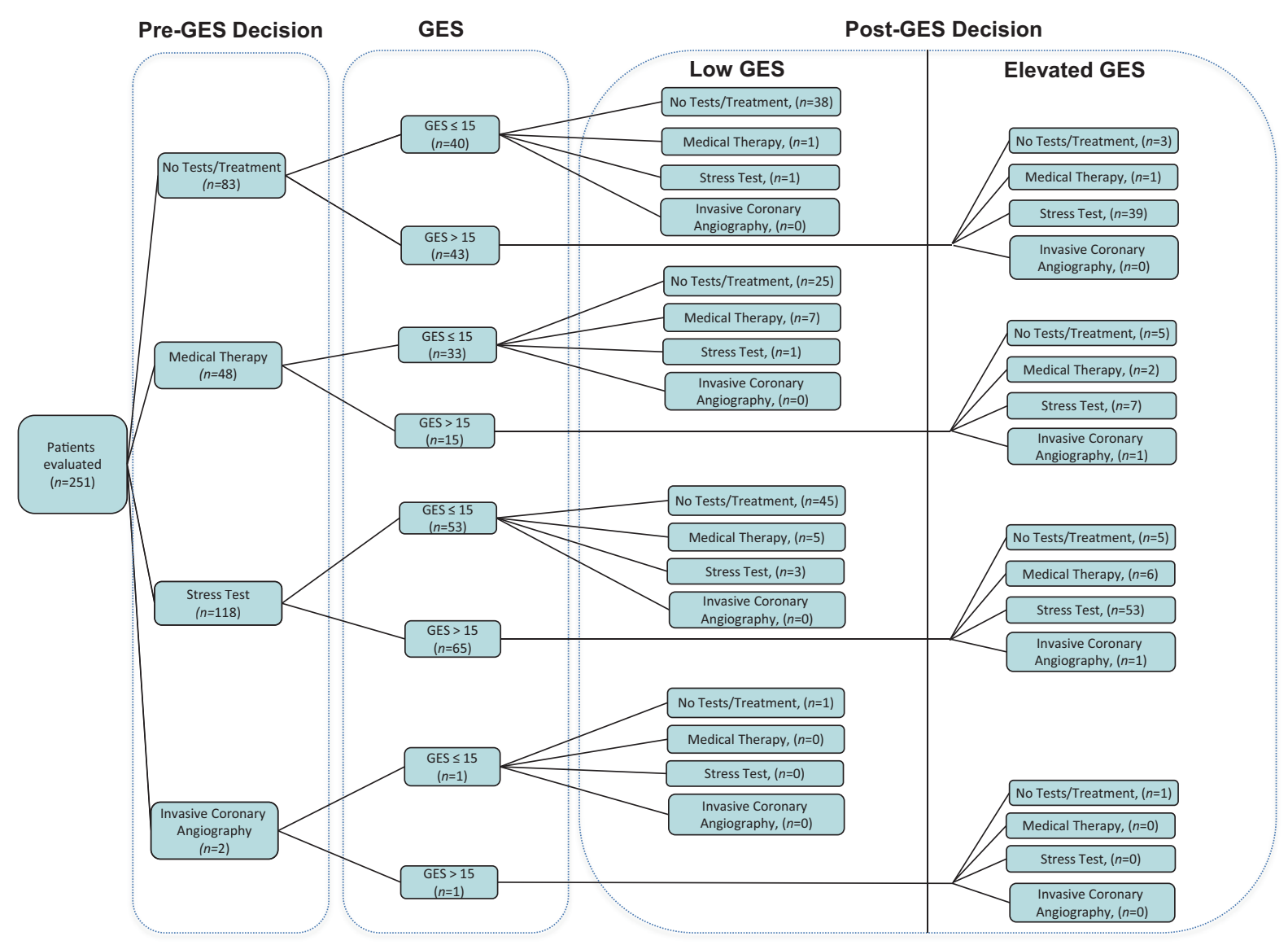

study, which demonstrated a $96 \%$ negative predictive value for patients with a low GES undergoing evaluation for obstructive CAD and a lack of untoward outcomes among this group during 6 months of follow-up. ${ }^{28}$ This level of diagnostic accuracy allows physicians to quickly, efficiently, and safely rule out cardiac causes among patients with a low GES and focus their workup on noncardiac causes of the patients' presenting symptoms. For patients with an elevated GES, the workup is more complex and suggests that the traditional referral pattern for additional cardiac evaluation and imaging may be more appropriate.

There were several limitations to the design and conduct of this study. First, the GES test is not intended for use in patients with known obstructive CAD or in patients with diabetes or systemic inflammatory diseases. Diabetic patients are at high risk of having underlying disease and thus are less likely to derive benefit from a "rule out" test. While the exclusion of patients with diabetes may limit the patient population indicated for the test, the pop- ulation eligible for GES testing includes more than 3 million patients per year who present to primary care practices in the United States for evaluation of symptoms suggestive of obstructive CAD. ${ }^{1}$

Second, the sample size was modest, with 251 patients evaluated in this prospective before/after GES cohort. Despite this modest size, the results were statistically significant in showing the incremental contribution of GES to clinical decision making. Third, since the primary objective of the study was to assess whether the use of the GES altered the clinicians' evaluations, we did not power the study to examine the association of the GES results with outcomes of further diagnostic testing. However, the $11 \%$ rate (6 of 56 patients) of positive stress tests in the patient cohort with an elevated GES is higher than the recently published figures from the large survey reported by Rozanski et al, ${ }^{13}$ which showed an $8 \%$ overall positive rate on MPI testing. Furthermore, only 1 patient in our trial went on for invasive cardiac angiography; this patient was in the elevated GES cohort and was found 
to have significant obstructive CAD. These last 2 findings are consistent with the confirmed ability of the GES to identify not only the likelihood of obstructive CAD but also the burden of the disease. $^{36}$

Fourth, while clinical outcomes were monitored for at least 30 days, it may be possible that adverse cardiac events occurred after this follow-up period. Previous evaluation of patients from the COMPASS and PREDICT validity studies have shown a very low rate of major adverse cardiovascular events over 6 and 12 months followup, respectively, among patients with low GES. ${ }^{28,37}$ In addition, in 2 clinical utility studies with GESdirected care involving 399 patients followed for a minimum of 6 months, only 1 patient (with an elevated GES) had a major adverse cardiovascular event. ${ }^{38}$ By comparison, several studies have shown adverse events associated with the use of contrast dye and radiation exposure from advanced noninvasive and invasive cardiac testing, including complications such as nausea and vomiting in $15 \%$ of patients and major adverse cardiovascular events in up to $1 \%$ of patients. ${ }^{23-25,39-41}$ Use of the GES to appropriately exclude the presence of obstructive CAD in these low- to intermediate-risk patients may help prevent these adverse events from cardiac imaging.

\section{Conclusions}

In the PREDICT and COMPASS trials, the noninvasive, genomic-based, personalized medicine test providing a GES was shown to have clinical validity in the assessment of obstructive CAD. Our study demonstrates the clinical utility of the GES test in the primary care setting. The use of the GES by primary care clinicians as part of the diagnostic workup may influence the clinical management of patients through improved risk stratification. By quickly, efficiently, and safely ruling out significant cardiac causes, the diagnostic focus may turn toward noncardiac causes of patients' presenting symptoms. Given current initiatives concentrating on eliminating inefficiencies in clinical care and improving the quality of patient care, the GES may represent a dynamic step forward for the daily dilemma primary care physicians face when evaluating stable, nonacute patients, especially women and those at low to intermediate risk who present with chest pain and related symptoms.
The authors thank Michael Conlin, MD, and Pamela Watson, APRN, for their invaluable contributions to patient recruitment and clinical data acquisition. The authors also thank Phil Beineke, Elsa Hewlett, and Lori Littleford for their attention to detail during patient monitoring and data analysis, as well as Joseph Ladapo, MD, PhD, for his thoughtful review of the manuscript.

\section{References}

1. Roger VI, Go A, Loyd-Jones DM, et al; American Heart Association Statistics Committee and Stroke Statistics Subcommittee. Executive summary: heart disease and stroke statistics-2012 update: a report from the American Heart Association. Circulation 2012;125:188-97.

2. Forrest C, Nutting P, Starfield B, Von Schrader S. Family physicians' referral decisions. J Fam Pract 2002;51:215-22.

3. Katerndahl DA. Panic plaques: panic disorder and coronary artery disease in patients with chest pain. J Am Board Fam Pract 2004;17:114-26.

4. Katerndahl DA, Realini P. Where do panic attack sufferers seek care? J Fam Pract 1995;40:237-43.

5. Chaitman BR, Bourassa MG, Davis K, et al. Angiographic prevalence of high-risk coronary artery disease in patient subsets (CASS). Circulation 1981;64: $360-7$.

6. Diamond GA, Forrester JS. Analysis of probability as an aid in the clinical diagnosis of coronary-artery disease. N Engl J Med 1979;300:1350-8.

7. Swap C, Nagurney J. Value and limitations of chest pain history in the evaluation of patients with suspected acute coronary syndromes. JAMA 2005;294: $2623-9$.

8. An exploratory report of chest pain in primary care. A report from ASPN. J Am Board Fam Pract 1990; $3: 143-50$.

9. Katerndahl DA, Trammell C. Prevalence and recognition of panic states in STARNET patients presenting with chest pain. J Fam Pract 1997;45:54-63.

10. Klinkman MS, Stevens D, Gorenflo DW. Episodes of care for chest pain: a preliminary report from MIRNET. Michigan Research Network. J Fam Pract 1994;38:345-52.

11. Cayley WE Jr. Diagnosing the cause of chest pain. Am Fam Physician 2005;72:2012-21.

12. Cheng VY, Berman DS, Rozanski A, et al. Performance of the traditional age, sex, and angina typicality-based approach for estimating pretest probability of angiographically significant coronary artery disease in patients undergoing coronary computed tomographic angiography: results from the multinational coronary CT angiography evaluation for clinical outcomes: an international multicenter registry (CONFIRM). Circulation 2011;124:2423-32, $1-8$.

13. Rozanski A, Gransar H, Hayes SW, et al. Temporal trends in the frequency of inducible myocardial isch- 
emia during cardiac stress testing: 1991 to 2009. J Am Coll Cardiol 2013;61:1054-65.

14. Gibbons RJ, Balady GJ, Bricker JT, et al. ACC/ACH 2022 guideline update for exercise testing: summary article. A report of the American College of Cardiology/American Heart Association Task Force on Practice Guidelines (Committee to Update the 1997 Exercise Testing Guidelines). J Am Coll Cardiol 2002;40:1531-40.

15. Gallagher MJ, Ross MA, Raff GL, Goldstein JA, O'Neill WW, O'Neill B. The diagnostic accuracy of 64-slice computed tomography coronary angiography compared with stress nuclear imaging in emergency department low-risk chest pain patients. Ann Emerg Med 2007;49:125-36.

16. Shaw LJ, Peterson ED, Shaw LK, et al. Use of a prognostic treadmill score in identifying diagnostic coronary disease subgroups. Circulation 1998;98: 1622-30.

17. Patel MR, Peterson ED, Dai D, et al. Low diagnostic yield of elective coronary angiography. N Engl J Med 2010;362:886-95.

18. IMV. IMV 2005 Nuclear Medicine Census Market Summary Report. Des Plaines, IL: IMV Medical Information Division; 2005.

19. IMV. IMV 2006 Cardiac Catheterization Lab Census Market Summary Report. Des Plaines, IL: IMV Medical Information Division; 2006.

20. IMV. IMV 2008 Present Practices \& Future Directions in Cardiac Imaging, The Cardiologist's Perspective 2008-2011. Des Plaines, IL: IMV Medical Information Division; 2008.

21. IMV. IMV 2004 Echocardiography Census Market Summary Report. Des Plaines, IL: IMV Medical Information Division; 2004.

22. Chen J, Einstein A, Fazel R, et al. Cumulative exposure to ionizing radiation from diagnostic and therapeutic cardiac imaging procedures: a populationbased analysis. J Am Coll Cardio 2010;56:702-11.

23. Einstein A, Moser KW, Thompson RC, Cerqueira MD, Henzlova MJ. Radiation dose to patients from cardiac diagnostic imaging. Circulation 2007;116: 1290-305.

24. Einstein AJ, Weiner SD, Bernheim A, et al. Multiple testing, cumulative radiation dose, and clinical indications in patients undergoing myocardial perfusion imaging. JAMA 2010;304:2137-44.

25. Rhee C, Bhan I, Alexander EK, Brunelli SM. Association between iodinated contrast media exposure and incident hyperthyroidism and hypothyroidism. Arch Intern Med 2012;172:153-9.

26. Elashoff MR, Wingrove JA, Beineke P, et al. Development of a blood-based gene expression algorithm for assessment of obstructive coronary artery disease in non-diabetic patients. BMC Med Genomics 2011; 4:26.

27. Rosenberg S, Elashoff MR, Beineke P, et al. Multicenter validation of the diagnostic accuracy of a blood-based gene expression test for assessing obstructive coronary artery disease in nondiabetic patients. Ann Intern Med 2010;153:425-34.

28. Thomas G, Voros S, McPherson J, et al. A blood based gene expression test for obstructive coronary artery disease tested in symptomatic non-diabetic patients referred for myocardial perfusion imaging: the COMPASS study. Circ Cardiovasc Genet 2013; 6:154-62.

29. Gibbons RJ, Abrams J, Chatterjee K, et al. ACC/ AHA 2002 guideline update for the management of patients with chronic stable angina-summary article: a report of the American College of Cardiology/ American Heart Association Task Force on practice guidelines (Committee on the Management of $\mathrm{Pa}$ tients With Chronic Stable Angina). J Am Coll Cardiol 2003;41:159-68.

30. Conover WJ. Practical nonparametric statistics. New York: John Wiley \& Sons; 1971:97-104.

31. Bowman MA, Neale AV. Researching together and improving practice outcomes. J Am Board Fam Med 2013;26:1-3.

32. McPherson JA, Davis K, Yau M, et al. Gene expression testing improves the diagnostic evaluation of patients presenting to the cardiologist with symptoms of suspected obstructive coronary artery disease: results from the IMPACT (Investigation of a Molecular Personalized Coronary Gene Expression Test on Cardiology Practice Pattern) trial. Crit Pathw Cardiol 2013;27:37-42.

33. Steinhart B, Thorpe KE, Bayoumi AM, Moe G, Januzzi JLJ, Mazer CD. Improving the diagnosis of acute heart failure using a validated prediction model. J Am Col Cardiol 2009;54:1515-21.

34. Lo S, Mumby PB, Norton J, et al. Prospective multicenter study of the impact of the 21-gene recurrence score assay on medical oncologist and patient adjuvant breast cancer treatment selection. J Clin Oncol 2010;28:1671-6.

35. Nystrom JS, Hornberger J, Varadhachary G, et al. Clinical utility of gene-expression profiling for tumor-site origin in patients with metastatic or poorly differentiated cancer: impact on diagnosis, treatment, and survival. Oncotarget 2012;3:620-8.

36. Wingrove JA, Daniels SE, Sehnert AJ, et al. Correlation of peripheral-blood gene expression with the extent of coronary artery stenosis. Circ Cardiovasc Genet 2008;1:31-8.

37. Rosenberg S, Elashoff MR, Lieu HD, et al. Whole blood gene expression testing for coronary artery disease in nondiabetic patients: Major adverse cardiovascular events and interventions in the PREDICT Trial. J Cardiovasc Transl Res 2012;2012:7.

38. Conlin MF, Herman LE, Wilson L, et el. The use of a personalized gene expression test to improve decision making in the evaluation of patients with suspected coronary artery disease. J Gen Intern Med 2012;27:S540-1. 
39. Fazel R, Krumholz HM, Wang Y, et al. Exposure to low-dose ionizing radiation from medical imaging procedures. N Engl J Med 2009;361:849-57.

40. Chen J, Einstein AJ, Fazel R, et al. Cumulative exposure to ionizing radiation from diagnostic and therapeutic cardiac imaging procedures: a population-based analysis. J Am Coll Cardiol 2010;56: 702-11.

41. Lange R, Hillis L. Diagnostic cardiac catheterization. Circulation 2003;107:e111-e3. 